Aim To successfully up-skill the hospice staff and to safely and effectively introduce SystmOne onto the IPU.

Approach used The introduction of SystmOne was seen as a major change for the IPU and hence a change management model was used to support this. The main areas addressed were process mapping, to identify areas where we could work more effectively and staff training. Staff training was of paramount importance as $26 \%$ of the IPU staff had no basic IT skills. This was given to those staff before embarking upon the SystmOne training.

The ward staff were all involved in the development of new IPU documentation and the review of nursing care plans.

The change has been anchored by the alteration in ward routine, staff job descriptions and the change in hospice policies. Outcomes

- Improved communication between departments and external organisations who use SystmOne

- Reduced amount of time spent completing documentation

- Improved documentation standards

- Reduction in patients being asked the same questions on several occasions

- More than one person/department being able to access the patient notes at any one time

- Elimination of time spent searching for patient notes

- Enhanced management of out of hours phone calls thereby improving clinical safety

- Improved audit and monitoring

Application to hospice practice We were one of the first IPU's to 'go-live' with Systmone and the improvements have been wide-reaching for both patients and staff.

Nicola Butterfield

Lead Nurse, IPU

Birmingham St Mary's Hospice

\section{P83 APPLYING NHS PRODUCTIVE SERIES MODEL TO HOSPICE CARE - RELEASING TIME TO CARE}

Cathryn Goodchild, Maggie Draper. St Luke's Hospice Plymouth, Plymouth, United Kingdom

\subsection{6/bmjspcare-2013-000591.105}

Introduction Productive Ward and Productive Community are a series of modules designed to deliver sustainable service improvement, and are well established in the NHS. We decided to adopt the model, fund the education and development and apply it to:

- Hospice Inpatient Unit

- Hospice Day Care provision

- Hospice Community Palliative Care team

- Hospice Lymphodema Service

Aims To use the series across the organisation to improve efficiency and consider processes in a systematic way using a tested methodology. This will lead to reduction in time wasted, duplication, frustrations etc and the aim is that the time saved can be re-invested in direct patient care. This involves a culture shift within the organisation to adopt new ways of working and is unique within the Hospice movement. The aim is to embed new improved ways of working across the organisation and to empower staff from the "ground up".

Methods
- External training delivered

- Steering group established

- Launch in different teams and establishment of champions

- Work streams to deliver 2 modules in first year :

- Knowing How We Are Doing - public display of information e.g.: response times, no of referrals, falls, staffing levels/ sickness rates/ caseload averages/RAG rating etc.

- Well Organised Working Environment /Wardreview of referral process and pathway, evaluating stock levels and standardisation of store cupboards/ clinician's bags etc.

Evaluation The Productive Series model encourages:

- Continuous evaluation and feedback to teams.

- Ownership of changes, sustaining improvement and embedding practice

- Empowering of staff across bands and settings to deliver the organisation's vision and release time to care.

Further information to follow regarding roll out, changes adopted and impact evaluated by October 2013

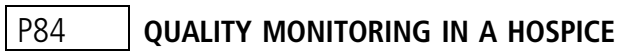

Pauline Flanagan, Michelle Roberts. Douglas Macmillan Hospice, Stoke-on-Trent, UK

10.1136/bmjspcare-2013-000591.106

Background/context Hospices are expected to quantify the quality of care and demonstrate outcome improvements ${ }^{1,2}$. There has been a change in emphasis from system and process to outcome $^{1-4}$. However, little hospice-specific guidance is available ${ }^{1-6}$.

The DMH produces quarterly quality monitoring (QM) reports and is seeing useful intelligence. Aim

- To produce hospice-specific intelligence

- To evidence that the hospice remains low risk.

Approach used QM is part of the hospice's well-established clinical governance function. Reports use monthly (\& rolling annual) data with published statistics (Office of National Statistics; National End of Life Care Intelligence Network).

Electronic patient-notes enable data collation in a way that was impossible with paper notes.

Hospice-specific outcome indicators have been developed to evidence compliance with outcome measures given in the NICE 2011 quality statements ${ }^{2}$ and the essential standards of quality and safety ${ }^{4}$.

Outcomes The hospice has robust evidence of the following:

- Number (\%) and diagnosis of patients receiving care

- Primary care aim

- Equity of access

- Impact of the 24/7 advice line

- Percentage of hospice

$\circ$ deaths of patients offered an ACP

- patients achieving their preferred place of care.

- Percentage of in-patients

$\circ$ on the LCP at the time of their death.

$\circ$ assessed for risks associated with moving and handling.

- experiencing minor/serious injury.

$\circ$ assessed for tissue viability within 6 hours of admission. 
- Waterlow score - an indication of patient frailty.

The DMH can demonstrate that twice as many EOL patients receiving care die at home as those not referred to the hospice.

\section{\begin{tabular}{|l|l}
\hline P85 DEVELOPING AUDIT IN A HOSPICE \\
\hline
\end{tabular}}

Amanda Gregory, Laura Myers. St. Catherine's Hospice, Crawley, West Sussex

10.1136/bmjspcare-2013-000591.107

The clinical audit group (CAG) at an independent hospice aims to motivate and encourage staff to undertake audit and to provide support and education for this activity. Many clinical staff have audit included in their job description. It was, however, observed that, while some staff regularly and competently carry out audit, some have not participated in audit and others find it difficult to conduct audit and report on their work, with variable knowledge of the theory and practice of audit. The CAG have, therefore, sought to implement a more robust audit registration and approval system alongside a programme of education to develop audit skills across the hospice in order to be able to produce credible and appropriate audit that demonstrates quality and areas for improvement. A set of resources to support audit was produced and made accessible to hospice staff electronically. A local step by step guide to registering an audit was produced. This included ensuring that CAG members review all audit registrations and recommendations for refinements are made before an audit is approved to start. The education team provides support to plan audits, including survey design, analysis and presentation of data. An audit of audits revealed that the quality of audits had improved (following implementation of some of these steps), while highlighting the a need to strengthen audit reporting, action plans and re-audit planning. A regular newsletter, highlighting audit activity within the organisation has been produced and audit activity has been reported in the general hospice newsletter. More recently, a questionnaire issued to the CAG members demonstrated gaps in knowledge and understanding, suggesting the need for further training to enable them to support their colleagues in undertaking audit. A programme of education has been designed to meet this need. The structure and membership of CAG is also being reviewed.

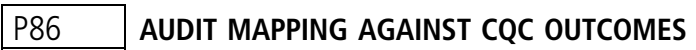

Jo Stanford, Ruth Phillips. Shooting Star CHASE, Surrey, England

\subsection{6/bmjspcare-2013-000591.108}

Audit mapping against CQC Outcomes

Introduction The Care Quality Commission (CQC) requires agencies to provide evidence that they are meeting the CQC outcomes. Clinical Governance is a framework through which organisations are accountable for continuously improving the quality of their services and safeguarding high standards of care. Aims The Clinical Governance Reporting Guidelines have been reviewed and restructured inline with the Care Quality Commission (CQC) outcomes. The purpose of this exercise was to streamline the audit reporting process inline with the CQC, make the audits more meaningful, and avoid duplication of work.

Methods Using the hospices existing Clinical Governance Audit Programme, where possible, each CQC outcome was allocated to an audit. Some audits were allocated more than one 'outcome'

Results Below is an example of how some of the audits are mapped against the CQC Outcomes.

\begin{tabular}{|c|c|c|c|c|}
\hline Category & Audit title & $\begin{array}{l}\text { Reference } \\
\text { number }\end{array}$ & $\begin{array}{l}\text { Frequency } \\
\text { of audit }\end{array}$ & CQC Outcome \\
\hline Involvement and Info & Peer - Activities & 1.1 & 6 monthly & $1 a, 1 b, 1 c, 1 f$ \\
\hline Safeguarding and Safety & Safeguarding & 3.7 & Quarterly & $4 b, 4 i, 7 a, 7 b$, \\
\hline \multirow{6}{*}{$\begin{array}{l}\text { Personalised Care, } \\
\text { treatment and support } \\
\text { Quality of management }\end{array}$} & Symptom & 2.4 & 6 monthly & $1 \mathrm{a}, 1 \mathrm{~b}, 1 \mathrm{c}, 4 \mathrm{~d}$ \\
\hline & Management & & & $4 \mathrm{e}, 4 \mathrm{k}, 4 \mathrm{w}, 9 \mathrm{a}$ \\
\hline & $A \& 1$ & 5.1 & Quarterly & $4 b, 11 a, 11 c$ \\
\hline & & & & $16 c, 20 a, 20 b$ \\
\hline & Complaints & 5.2 & Quarterly & $16 a, 17 a, 17 e$ \\
\hline & & & & $20 \mathrm{f}, 20 \mathrm{~h}, 20 \mathrm{i}$ \\
\hline
\end{tabular}

Discussion The children's hospice audit programme meets and provides evidence for many of the CQC Outcomes. Evidence for those the outcomes that are not met, can be provided in various other ways.

Conclusion This mapping exercise has been helpful in identifying how the hospice was already providing evidence for the CQC, but also helped to identify any shortfalls. Some audits have been reviewed to ensure that they serve a purpose and also can provide evidence for the CQC.

\section{P87 MAY I?: AUDIT OF DOCUMENTATION OF CONSENT FOR CARE}

Helen Birch, Catherine Baldry, Karen Groves, Shirley Balmer. Queenscourt Hospice, Southport, UK

\subsection{6/bmjspcare-2013-000591.109}

Aims Consent to valid treatment and care is central in all forms of healthcare: patients have a fundamental, legal and ethical right to determine what happens to them. Hospice at Home works with community services to fill gaps in end of life care for patients whose preferred place of care is home. Care is provided predominantly by health care assistants, and a few registered nurses (RN's). RN's receive consent training in their nursing studies. The aim was that all hospice at home evaluations should clearly state 'consent status documented for all care interventions given'. Many patients are unconscious and unresponsive when care interventions occur. Hospice policy states 'Consent status must be documented for all personal and invasive procedures'. It was difficult to see at a glance if consent status was recorded in the care plan.

Method A retrospective (random) audit of 204 care plans was performed to determine if consent status for personal care was documented. Confidentiality was maintained at all times. $77 \%$ records completed by HCAs and $23 \%$ by RNs

Result Cycle $1-74 \%$ of care plans had consent status recorded. $26 \%$ records had no evidence of consent status documented. Further analysis identified only $37 \%$ of RNs had recorded consent status. Training needs were identified and implemented in various ways to include Consent, Mental Capacity Act (2005), and Record keeping. Cycle 2 Re- audited 6 months later using same criteria and data collection tools. - 93\% of care plans had consent status recorded for personal care, improvement in the number of RN's recording consent status- 77\% Results Care plans now checked daily to identify staff not adhering to policy. The need to replicate audit in other areas of the Palliative Care 